\title{
The Functional Independence Measure: a comparative study of clinician and self ratings
}

\author{
N Grey BA, P Kennedy MSc C Psychol \\ Department of Clinical Psychology, National Spinal Injuries Centre, Stoke Mandeville \\ Hospital, Aylesbury, Bucks HP21 8AL, UK.
}

\begin{abstract}
In recent years the Functional Independence Measure has emerged as a standard assessment instrument for use in rehabilitation and therapy programmes for disabled persons, including those with spinal cord injury (SCI). This measure was devised to be rated by a clinician familiar with the patient. We studied 40 spinal cord injury patients who were rated on the FIM by a clinician within the 6 weeks prior to discharge, and who then rated themselves on the FIM at one month post discharge. There was a strong correlation between the differently rated scores. This suggests that the FIM can be given to patients as a self-report questionnaire, thus reducing time of assessment and increasing assessment potential.
\end{abstract}

Keywords: spinal cord injury; Functional Independence Measure (FIM); self-report.

\section{Introduction}

It has long been recognised that the functional assessment documentation of severity of patient disability can be useful in identifying particular problems in rehabilitation, to quantify patient progress, and to establish priorities when devising therapy programmes. An early measure of the level of functional independence, still being used, is the Barthel Index. ${ }^{1}$ This was designed to measure improvement during inpatient rehabilitation through weighted scales of self care (feeding, bathing, toileting, dressing, bladder and bowel management) and mobility (transfers, ambulation, stair climbing). It has been used in numerous contexts, including rehabilitation of stroke patients ${ }^{2}$ and those with spinal cord injury (SCI). ${ }^{3}$

However a drawback of the Barthel Index and other scales such as the Katz Index of Activities of Daily Living, ${ }^{4}$ the PULSES profile, ${ }^{5}$ and the Incapacity Status Scale ${ }^{6}$ is that communicative and cognitive deficits are not included. Thus it is possible for an individual to score well on these scales without being able to live alone or participate socially. In order to attempt to meet these needs and to provide a uniform way to communicate about disability and evaluate the rehabilitation progress and the outcome of patients with disabilities, the Task Force to Develop a Uniform Data System for Medical Rehabilitation developed the Functional Independence Measure (FIM). ${ }^{7}$

The FIM measures six areas of functioning, subsuming but extending the Barthel Index: self care (feeding, grooming, bathing, dressing upper body, dressing lower body, toileting); sphincter control (bladder management, bowel management); mobility (transfers to and from bed/chair, toilet, tub-shower); locomotion (walk/chair, stairs); communication (comprehension, expression); and social cognition (social interaction, problem solving, memory). It was designed to include a minimum of items and to be usable 'by any trained clinician, regardless of discipline'. It allows clinicians and researchers to track patients by measuring performance over time.

Originally each scale was scored on 4 levels but a revised version has been developed which scores each scale on 7 levels, from 7 being complete independence to 1 being total assistance. ${ }^{8}$ The reliability of this version has been tested by two or more pairs of clinicians assessing each of 263 patients. This gave an intraclass correlation co- 
efficient of $0.95 .^{9}$ Face validity of the FIM was evaluated by asking clinicians specific questions regarding difficulty of understanding ( $88 \%$ had no difficulty), unnecessary items $(97 \%$ felt that there were no unnecessary items), and items which should be added ( $83 \%$ felt no need for extra items). The FIM has been adopted by the American Spinal Injury Association and the International Medical Society of Paraplegia as the standard measure of functional independence in persons with SCI.

In an assessment of persons with multiple sclerosis (MS) ${ }^{10}$ the FIM correlated highly with the Barthel Index and the Incapacity Status Scale, and was found to be the most predictive of the MS patients' physical care needs, being the most precise about degrees of assistance. Recently the FIM has been used successfully to provide a functional assessment of patients in various clinical settings including stroke, ${ }^{11}$ AIDS, ${ }^{12}$ cancer, ${ }^{13}$ and SCI.$^{14}$ However in the case of lower-limb amputees the FIM was not particularly good at predicting rehabilitation outcomes. ${ }^{15}$ Furthermore it has been shown that the social cognition scales of the FIM cannot be used as a substitute for comprehensive neuropsychological assessment in patients with SCI. ${ }^{16}$ On balance, though, it appears that the FIM provides a good functional assessment of patients.

The FIM was designed to be completed by trained clinicians but a self-report version ${ }^{17}$ has been used in a shortened form for patients with SCI. ${ }^{18}$ This study collected data by clinician-client phone interviews with appropriate follow up questioning, whereas our study relies entirely on self report. The advantages of a self-rated FIM are that it will reduce time taken to make an assessment and increase assessment potential by allowing easier follow up postdischarge.

This study investigates the reliability of using the complete FIM as a self-report postal questionnaire without the need for any clinician to be present or for detailed instruction to be given. By comparing clinician ratings pre-discharge and self report post-discharge a significant correlation between the scores is expected. Implications for further research are discussed.

\section{Methods}

\section{Sample}

Forty patients were studied while in hospital and post-discharge. The inclusion criteria for the study were that the patients should be aged 16-65 years and have experienced traumatic SCI. The average age of the patients at time of injury was 29.6 years $(\mathrm{SD}=9.57)$, with a range of $17-54$. Eightyfive percent of the patients were male. Cause of injuries in descending order were road traffic accidents $45 \%$, falls $25 \%$ (50\% domestic, $50 \%$ industrial), sports injuries $22.5 \%$, other organic cause $7.5 \% ; 32.5 \%$ were tetraplegic and $67.5 \%$ were paraplegic. Average time post-injury upon discharge was 24.75 weeks $(S D=8.57)$ with a range 7-49. Average time between ratings under comparison was 7.25 weeks $(\mathrm{SD}=1.93)$ with a range $4-10$. The compliance rates were $90 \%$ pre-discharge and $67 \%$ for the post-discharge postal questionnaire.

\section{Materials}

The Functional Independence Measure as developed by the Task Force to Develop a Uniform Data System for Medical Rehabil-

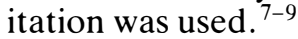

\section{Procedure}

The patients were rated every 6 weeks from admission to discharge on the FIM. The FIM was rated by a staff nurse or ward sister who knew the patient's condition well. Training of staff was informal as formal training was not available in the UK. Upon discharge each subject was sent the FIM at regular intervals in order to track their progress and be kept informed of their situation and problems. The first FIM was completed one month post-discharge. The self-report FIM consists purely of the original scoring sheet with minimal instructions, ie $7=$ complete independence; $6=$ modified independence (use of a device); $2=$ maximal assistance (do $25 \%$ yourself); $1=$ total assistance (do $0 \%$ yourself). There was no interview or further help or instructions.

The last clinician-report score prior to 
discharge and self-report score at one month post-discharge on the FIM from the 40 patients within this study were compared and contrasted.

Ethical approval and consent were obtained for this study.

\section{Results}

There was no significant difference between the differently rated total FIM scores $(t=0.279, p=0.781)$. The average predischarge clinician-rated FIM score $=104.9$ $(\mathrm{SD}=20.2)$, with a range 51-125. The average post-discharge self-rated FIM score $=106.3(\mathrm{SD}=23.7)$, with a range 37-126. Importantly for our hypothesis the two scores were strongly correlated $(r=$ $0.828)$, significant at the $p<0.0001$ level (Table I).

In order to determine whether the correlation of the total FIM scores was an artefact of summing the many different component scales, these scales were also independently analysed, finding no significant differences between the differently rated scores on any of the scales. Comparing the clinician-rated and self-rated scores strong correlations for each of the scales of self care, sphincter control, mobility and locomotion were found, but no correlation for the scales of communication or social cognition.

\section{Discussion}

The results of this study demonstrate a clinically significant relationship between the final pre-discharge clinician-rated FIM scores and the one month post-discharge self-rated FIM scores. The correlation of $r=0.828$ compares favourably to the interrater correlation of $r=0.86$ found with the original FIM, ${ }^{7}$ but is lower than the correlation of $r=0.95$ with the revised 7-level FIM. ${ }^{9}$

The results from the component scales, showing no significant differences between the clinician-rated and self-rated scores on any scale, demonstrate that the correlation between the differently rated scores for the total FIM is not merely an experimental artefact but that each component helps build an overall picture. The high correlations between the differently rated scales of self care, sphincter control, mobility and locomotion indicate that they could be used as reliable self-report measures separately and individually if necessary. The lack of

Table I Mean scores, standard deviations and correlations for clinician-rated and self-rated FIM scales

\begin{tabular}{lcccc}
\hline FIM scale & $\begin{array}{c}\text { Clinician- } \\
\text { rated }\end{array}$ & $\begin{array}{c}\text { Self- } \\
\text { rated }\end{array}$ & $\begin{array}{c}\text { Correlation } \\
\text { of ratings }\end{array}$ & $p$ \\
\hline Self care $/ 42$ & $\begin{array}{c}34.625 \\
(10.4)\end{array}$ & $\begin{array}{c}35.475 \\
(10.9)\end{array}$ & 0.841 & 0.0001 \\
Sphincter control $/ 14$ & $\begin{array}{c}10.850 \\
(3.75)\end{array}$ & $\begin{array}{c}11.325 \\
(4.13)\end{array}$ & 0.710 & 0.0001 \\
Mobility $/ 21$ & 17.325 & 16.900 & 0.733 & 0.0001 \\
& $(5.67)$ & $(6.28)$ & & \\
Locomotion $/ 14$ & 8.125 & 9.150 & 0.454 & 0.0032 \\
Communication $/ 14$ & $(2.05)$ & $(3.53)$ & & $\mathrm{NS}$ \\
Social cognition $/ 21$ & 13.575 & 13.475 & 0.029 & $\mathrm{NS}$ \\
Total & $(1.45)$ & $(1.91)$ & & 0.085 \\
& 20.275 & 20.175 & 0.085 & 0.0001 \\
\hline
\end{tabular}

Standard deviations in parentheses. 
correlation in the communication and social cognition scales arises due to a ceiling effect ( $92 \%$ of subjects and $88 \%$ of clinicians reported a maximum score on communication; and $75 \%$ of subjects and $73 \%$ of clinicians reported a maximum score on social cognition). This would also tend to support Davidoff et al's claim that these scales cannot be used as a substitute for thorough neuropsychological assessment in the identification of cognitive deficits. ${ }^{16}$ This does not invalidate use of the total FIM as a self-report questionnaire since there were no significant differences between the differently rated scores on these scales.

Overall, it does appear that use of the FIM as a self-rated questionnaire is reliable. It also offers many advantages compared to a clinician-rated measure. In Muecke et al's study of lower-limb amputees it is stated that the 'hospital did not routinely determine follow-up FIM because of manpower limitations'. ${ }^{15}$ A self-rated FIM would, in this case, have enabled more complete data collection and proper evaluations of follow-up therapy programmes. The brevity and ease of administration of this measure lends itself to numerous clinical situations where a continued functional assessment is called for, and also to research where collection of data could otherwise prove awkward and time consuming. The previously used self-rated FIM was conducted through interview with a clinician, ${ }^{18}$ whereas this study shows that such a procedure is unnecessary. In addition, this study has shown that the complete FIM can be used rather than a shortened version, which allows direct comparison with earlier clinician-rated scores.

However, three points must be addressed. Firstly, in order to complete the FIM the patient/subject must be functioning highly enough on the social cognition and communication scales of the measure. This can result in a ceiling effect on these scales as seen in this study, and will also tend to preclude use of a self-report FIM on pa- tients who have suffered long-term cognitive damage. The completion of the FIM by the carers of such patients, without clinician interview or supervision, is a possible avenue of interest that would require further investigation. Secondly, the SCI patients in our study had all passed through a goal planning/needs assessment system prior to discharge which raises the patients' awareness of their own needs and abilities, and hence perhaps makes them more able than most to complete the FIM. This would indicate perhaps the need for other similar studies on patients who have not passed through such a system, and on patients other than those with SCI. Thirdly, it is possible that the lack of significant differences may arise due to the opposite and equalising effects of the differences in rater, type of assessment, setting of assessment, and time of assessment. Further investigations comparing clinical observation, clinician-client interview and self report at a fixed point in time, in both hospital and home settings, would help elucidate this problem.

Thus, whilst not wishing to dismiss these issues, it seems that with some prior thought by the clinician or researcher about the relevance of a self-report FIM, it can become a useful tool. It is not suggested that this replaces clinical observation but that it can be used profitably in conjunction, and can help by reducing the time of assessment and increasing long-term assessment potential. This could be of particular interest and use for rehabilitation programmes in those spinal centres with limited resources, for inpatient review and outpatient follow up.

\section{Acknowledgements}

The authors would like to acknowledge the support of the Spinal Amenity Research Fund, $\mathrm{Dr} \mathrm{H}$ L Frankel, Mr B Gardner, Mr I Nuseibeh, Dr J Silver and patients who willingly cooperated.

\section{References}

1 Maloney FI, Barthel DW (1965) Functional evaluation: Barthel Index. MD State Med J 14: 61-65.

2 Granger CV, Dewis LS, Peters NC, Sherwood CC, Barrett JE (1979) Stroke rehabilitation: analysis of 
repeated Barthel Index measures. Arch Phys Med Rehabil 60: 14-17.

3 Alfred WG, Fuhrer MJ, Rossi CD (1987) Vocational development following severe spinal cord injury: a longitudinal study. Arch Phys Med Rehabil 68: 854-857.

4 Katz S, Ford AB, Moskowitz RW (1963) Studies of illness in the aged. The index of ADL: a standardized measure of biological and psychological function. JAMA 185: 914-919.

5 Granger CV, Albrecht GL, Hamilton BB (1979) Outcome of comprehensive medical rehabilitation: measurement by PULSES profile and Barthel Index. Arch Phys Med Rehabil 60: 145-154.

6 International Federation of Multiple Sclerosis Societies (1985) MRD. Minimal Record of Disability for Multiple Sclerosis. National Multiple Sclerosis Society, New York.

7 Hamilton BB, Fuhrer MJ, editors (1987) In: Rehabilitation Outcomes: Analysis and Measurement. Brooks, Baltimore: $137-147$

8 Hamilton BB, Granger CV (1990) Guide for the Use of the Uniform Data Set for Medical Rehabilitation. Research Foundation of State University of New York, Buffalo NY.

9 Hamilton BB, Laughlin JA, Granger CV, Kayton RM (1991) Interrater agreement of the seven level Functional Independence Measure. Arch Phys Med Rehabil 72, 790.

10 Granger CV, Cotter AC, Hamilton BB, Fiedler RC, Hens MM (1990) Functional assessment scales: a study of persons with multiple sclerosis. Arch Phys Med Rehabil 71: 870-875.

11 Wilson DB, Houle DM, Keith RA (1991) Stroke rehabilitation: a model predicting return home. West J Med 154: 587-590.

12 O'Dell MW, Crawford A, Bohi ES, Bonner FJ Jr (1991) Disability in persons hospitalized with AIDS. Am J Phys Med Rehabil 70: 91-5.

13 O'Toole DM, Golden AM (1991) Evaluating cancer patients for rehabilitation potential. West J Med 155: 384-387.

14 Menter RR, Whiteneck GG, Charlifue SW, Gerhart K, Solnick SJ, Brooks CA et al (1991) Impairment, disability, handicap and medical expenses of persons ageing with spinal cord injury. Paraplegia 29: 613-619.

15 Muecke L, Shekar S, Dwyer D, Israel E, Flynn DP (1992) Functional screening of lower-limb amputees: a role in predicting rehabilitation outcome? Arch Phys Med Rehabil 73: 851-858.

16 Davidoff GN, Roth EJ, Haughton JS, Ardner MS (1990) Cognitive dysfunction in spinal cord injury patients: sensitivity of the Functional Independence Measure subscales vs neuropsychologic assessment. Arch Phys Med Rehabil 71: 326-329.

17 Smith P, Hamilton BB, Granger CV (1990) FIM Decision Tree: The Fone FIM. Research Foundation of State University of New York, Buffalo NY.

18 Fuhrer MJ, Rintala DH. Hart KA, Clearman R, Young ME (1992) Relationship of life satisfaction to impairment, disability, and handicap among persons with spinal cord injury living in the community. Arch Phys Med Rehabil 73: 552-557. 